09

\title{
Люминофор ближнего и коротковолнового ИК-диапазона на основе ниобата литий-лантана со структурой кубического граната
}

\author{
(C) Я.В. Бакланова, О.А. Липина, Л.Г. Максимова, И.В. Бакланова, \\ А.Ю. Чуфаров, А.П. Тютюнник, В.Г. Зубков
}

Институт химии твердого тела УрО РАН, Екатеринбург, Россия

E-mail: baklanovay@ihim.uran.ru

\begin{abstract}
Активированные неодимом и гольмием кубические гранаты $\mathrm{Li}_{6} \mathrm{CaLa}_{2} \mathrm{Nb}_{2} \mathrm{O}_{12}: \mathrm{Nd}^{3+}, \mathrm{Ho}^{3+}$ получены твердофазным методом синтеза. Установлены основные закономерности изменения люминесцентных свойств твердых растворов $\mathrm{Li}_{6} \mathrm{CaLa}_{2} \mathrm{Nb}_{2} \mathrm{O}_{12}: \mathrm{Nd}^{3+}, \mathrm{Ho}^{3+}$ в видимом, ближнем и коротковолновом инфракрасном диапазоне при возбуждении лазерным излучением с длиной волны $808 \mathrm{~nm}$. Предложен механизм передачи энергии между активными центрами, предполагающий участие ионов $\mathrm{Nd}^{3+}$ в качестве сенсибилизаторов люминесценции ионов $\mathrm{Ho}^{3+}$. Низкая фононная энергия, высокая интенсивность люминесценции в диапазоне 2.0-3.0 $\mu \mathrm{m}$ и слабая апконверсионная люминесценция в области 450-780 $\mathrm{nm}$ позволяют рассматривать кубические ниобаты лития-лантана $\mathrm{Li}_{6} \mathrm{CaLa}_{2} \mathrm{Nb}_{2} \mathrm{O}_{12}: \mathrm{Nd}^{3+}, \mathrm{Ho}^{3+}$ в качестве перспективных люминофоров коротковолнового инфракрасного диапазона.
\end{abstract}

Работа выполнена в соответствии с государственным заданием ИХТТ УрО РАН.

DOI: 10.21883/FTT.2019.05.47603.10F

\section{1. Введение}

В настоящее время инфракрасное (ИК) излучение используется в различных областях, таких как биология, медицина, при создании систем дистанционного зондирования, оптической связи и передачи информации. Важным параметром, определяющим сферу применения материалов, люминесцирующих в ИК-области спектра, является длина волны излучения. Так, например, использование в волоконно-оптических линиях связи излучения с длинами волн 1.3 и $1.55 \mu \mathrm{m}$ в основном обусловлено характеристиками поглощения светонесущего элемента - оптического волокна $[1,2]$. Диапазон $2-3 \mu \mathrm{m}$ ограничен для приложений оптической связи из-за высокого поглощения воды в данной спектральной области, но подходит для применения в медико-биологических целях [3-5]. Традиционно излучение в указанном диапазоне достигается за счет $4 f-4 f$-переходов в ионах $\mathrm{Ho}^{3+}$, $\mathrm{Er}^{3+}$ и $\mathrm{Tm}^{3+}$ [3-7]. Однако напрямую перечисленные ионы не могут быть эффективно возбуждены серийными лазерными диодами с длиной волны излучения 808 или $980 \mathrm{~nm}$. Для достижения интенсивной эмиссии в коротковолновой ИК-области в качестве сенсибилизатора используют ионы $\mathrm{Nd}^{3+}$ или $\mathrm{Yb}^{3+}$, для которых вероятность поглощения излучения при 808 и $980 \mathrm{~nm}$ соответственно, значительно выше. Введение перечисленных редкоземельных ионов в оптическую матрицу обычно производят непосредственно при синтезе соединений путем добавления в исходную смесь соответствующих реактивов. Однако в ряде случаев существует чрезвычайная зависимость спектрально-люминесцентных свойств люминофоров даже от следовых концентраций лантаноидов, которые могут содержаться в виде примесей в используемых реактивах, в том числе и квалификации „ос.ч.“. Ранее было обнаружено, что следовые количества ионов $\mathrm{Er}^{3+}$ и $\mathrm{Tm}^{3+}$, не превышающие $10^{-5}-10^{-6} \mathrm{wt} . \%$, являются причиной возникновения ап-конверсии в видимом спектральном диапазоне в кристаллах $\mathrm{YVO}_{4}: \mathrm{Nd}^{3+}$ и $\mathrm{KGd}\left(\mathrm{WO}_{4}\right)_{2}$ [8,9]. Кроме того, для ряда германиевых соединений, допированных неодимом и гольмием, показано, что максимальная интенсивность люминесценции в диапазоне длин волн $2-3 \mu \mathrm{m}$ наблюдается при следовых концентрациях ионов $\mathrm{Ho}^{3+}$ [10-13]. Аналогично, небольшое содержание ионов $\mathrm{Tm}^{3+}(0.001-0.025$ at.\%) является достаточным для эффективной сенсибилизации ионов $\mathrm{Er}^{3+}$ и заметно увеличивает интенсивность эмиссии при $2.7 \mu \mathrm{m} \mathrm{в} \mathrm{Y}_{3} \mathrm{Al}_{5} \mathrm{O}_{12}: \mathrm{Er}^{3+}, \mathrm{Tm}^{3+}[14]$, и, напротив, концентрация $\mathrm{Pr}^{3+}$ более $10^{-4}$ mass.\% приводит к заметному уменьшению интенсивности и длительности люминесценции стоксовых ИК-полос излучения ионов $\mathrm{Nd}^{3+}$ в диапазоне $0.86-1.40 \mu \mathrm{m}$ в известном ИК-люминофоре $\mathrm{YPO}_{4}: \mathrm{Nd}^{3+}[15]$.

Соединения со структурой граната, допированные ионами лантаноидов, являются одними из наиболее широко исследуемых материалов для различных оптических приложений. Для тетрагонального граната $\mathrm{Li}_{7} \mathrm{La}_{3} \mathrm{Hf}_{2} \mathrm{O}_{12}: \mathrm{Nd}^{3+}$ обнаружена ИК-люминесценция, связанная с присутствием следовых количеств гольмия [16]. В дополнение к люминесценции при длинах волн 1.0-1.8 $\mathrm{m}$ показано усиление ИК-эмиссии в области $2.0-3.0 \mu \mathrm{m}$, что объясняется эффективным переносом энергии между ионами $\mathrm{Nd}^{3+}$ и $\mathrm{Ho}^{3+}$. В данной работе исследованы оптические свойства новых ИК-люминофоров $\mathrm{Li}_{6} \mathrm{CaLa}_{2} \mathrm{Nb}_{2} \mathrm{O}_{12}: \mathrm{Nd}^{3+}, \mathrm{Ho}^{3+}$ со структурой кубического граната. Поскольку для ниобатов характерны низкие значения фононной энергии решетки $[17,18]$, не превышающие $800 \mathrm{~cm}^{-1}$, эти соединения могут рассматриваться как перспективные люминофоры ближнего и коротковолнового ИК-диапазона. 


\section{2. Методика эксперимента}

Твердые растворы $\mathrm{Li}_{6} \mathrm{CaLa}_{2-x-y} \mathrm{Nd}_{x} \mathrm{Ho}_{y} \mathrm{Nb}_{2} \mathrm{O}_{12}$ $\left(x=2.7 \cdot 10^{-5}-0.1 ; y=4 \cdot 10^{-8}-0.05\right)$ были получены твердофазным методом. В качестве исходных реагентов использовали $\mathrm{La}_{2} \mathrm{O}_{3} \quad(99.99 \%), \quad \mathrm{Nd}_{2} \mathrm{O}_{3} \quad(99.99 \%)$ и $\mathrm{Ho}_{2} \mathrm{O}_{3}$ (99.99\%), предварительно отожженные при $900^{\circ} \mathrm{C}$ в течение $5 \mathrm{~h}, \mathrm{Nb}_{2} \mathrm{O}_{5}(99.9 \%), \mathrm{CaCO}_{3}(99.9 \%)$ и $\mathrm{Li}_{2} \mathrm{CO}_{3}(99.9 \%)$ с $10 \%$ избытком с учетом летучести лития при отжиге. Смесь исходных компонентов, взятых в стехиометрических количествах, тщательно перетирали и отжигали при температуре $700^{\circ} \mathrm{C}$ в течение $6 \mathrm{~h}$, далее прессовали в таблетки и обсыпали исходной смесью. Отжиг проводили при $900^{\circ} \mathrm{C}$ при $12-15 \mathrm{~h}$ с промежуточным перетиранием на воздухе. Оксиды лантана и неодима содержали следовые примеси гольмия, которые были определены с помощью масс-спектрометрии с индуктивно связанной плазмой на приборе Perkin Elmer Elan 9000 ICP-MS6: $1.2 \cdot 10^{-3}$ mass $\% \mathrm{Nd}^{3+}$ и $2 \cdot 10^{-6}$ mass $\% \mathrm{Ho}^{3+}$ в $\mathrm{La}_{2} \mathrm{O}_{3}$, $8 \cdot 10^{-3}$ mass $\% \mathrm{La}^{3+}$ и $4 \cdot 10^{-4}$ mass $\% \mathrm{Ho}^{3+}$ в $\mathrm{Nd}_{2} \mathrm{O}_{3}$. Поскольку содержание гольмия в реактиве $\mathrm{La}_{2} \mathrm{O}_{3}$ меньше, чем в $\mathrm{Nd}_{2} \mathrm{O}_{3}$, то по мере замещения лантана неодимом концентрация $\mathrm{Ho}^{3+}$ в $\mathrm{Li}_{6} \mathrm{CaLa}_{2-x-y} \mathrm{Nd}_{x} \mathrm{Ho}_{y} \mathrm{Nb}_{2} \mathrm{O}_{12}$ увеличивается с $y=4 \cdot 10^{-8}$ до $y=4.5 \cdot 10^{-7}$. В твердые растворы с постоянным содержанием неодима $\mathrm{Li}_{6} \mathrm{CaLa}_{1.975-y} \mathrm{Nd}_{0.025} \mathrm{Ho}_{y} \mathrm{Nb}_{2} \mathrm{O}_{12}$ гольмий вводили специально в заданных количествах $y=0.025$ и $y=0.05$.

Рентгенофазовый анализ синтезированных соединений осуществляли с помощью дифрактометра STADI-P (STOE), оснащенного линейным позиционно-чувствительным детектором. Съемка проводилась в $\mathrm{Cu} K_{\alpha 1}$ излучении в интервале углов $2 \theta 5-120^{\circ}$ с шагом $0.02^{\circ}$. В качестве внешнего стандарта использовали поликристаллический кремний с параметром элементарной ячейки $a=5.43075(5) \AA \AA$. Идентификация фаз проведена с использованием картотеки PDF2 (ICDD, 2016). Уточнение кристаллических структур соединений проведено методом полнопрофильного анализа Ритвельда с использованием программного пакета GSAS $[19,20]$.

Спектр комбинационного рассеяния света (КРС) соединения $\mathrm{Li}_{6} \mathrm{CaLa}_{2-x-y} \mathrm{Nd}_{x} \mathrm{Ho}_{y} \mathrm{Nb}_{2} \mathrm{O}_{12}\left(x=2.7 \cdot 10^{-5}\right.$; $\left.y=4 \cdot 10^{-8}\right)$ был записан на спектрометре RENISHAW-1000 $(\lambda=532 \mathrm{~nm}, \mathrm{Nd}$ :YAG, $P=25 \mathrm{~mW})$. Спектры поглощения регистрировали в диапазоне длин волн $200-1350 \mathrm{~nm}$ с помощью спектрофотометра UV-3600 (Shimadzu) с использованием $\mathrm{BaSO}_{4}(99.9 \%)$ в качестве эталона.

Спектры люминесценции в диапазоне длин волн от 1 до $3 \mu \mathrm{m}$ получены методом синхронной съемки с использованием монохроматора МДР-204 (90 геометрия, дифракционная решетка $300 \mathrm{lines} / \mathrm{mm}$, оптический затвор, осуществляющий модуляцию с частотой $200 \mathrm{~Hz}$, оптический фильтр ИКС 7) и $\mathrm{PbS}$ фотосопротивления (ЛОМО-Фотоника). В качестве внешнего источника возбуждения использовался диодный лазер $\left(\lambda_{\mathrm{ex}}=808 \mathrm{~nm}\right.$, $P=150 \mathrm{~mW}$, KLM-H808-120-5, ФТИ-Оптроник). Мощность лазерного излучения контролировали прибором
13 PEM 001 (Melles Griot). Для регистрации спектров апконверсионной люминесценции использовали охлаждаемый детектор H10720-01 (Hamamatsu). Кинетику распада возбужденного состояния гольмия ${ }^{5} I_{7}$ регистрировали с использованием осциллографа TDS-3052 (Tektronix). Длительность возбуждающих импульсов варьировалась от $25 \mathrm{~ns}$ до $100 \mathrm{~ns}$ при частотах повторения $100 \mathrm{~Hz}-5 \mathrm{kHz}$. Все вышеуказанные оптические измерения проводились при комнатной температуре.

\section{3. Экспериментальные результаты и их обсуждение}

Согласно данным рентгеновской дифракции, твердые растворы $\mathrm{Li}_{6} \mathrm{CaLa}_{2-x-y} \mathrm{Nd}_{x} \mathrm{Ho}_{y} \mathrm{Nb}_{2} \mathrm{O}_{12}$ $\left(x=2.7 \cdot 10^{-5}-0.1 ; \quad y=4 \cdot 10^{-8}-0.05\right) \quad$ кристаллизуются в кубической сингонии (пространственная группа $I a-3 d, Z=8)$. Все дифракционные рефлексы рентгенограмм исследуемых соединений соответствуют кубической фазе $\mathrm{Li}_{6} \mathrm{CaLa}_{2} \mathrm{Nb}_{2} \mathrm{O}_{12}$ (FIZ ICSD card № 161386), никаких дополнительных пиков от промежуточных фаз или примесей, типичных при твердофазном синтезе, таких как ниобат лития-лантана $\mathrm{La}_{2} \mathrm{LiNbO}_{6}$ или оксид лантана $\mathrm{La}_{2} \mathrm{O}_{3}$ не обнаружено (рис. 1). Структура кубического граната $\mathrm{Li}_{6} \mathrm{CaLa}_{2} \mathrm{Nb}_{2} \mathrm{O}_{12}$ состоит из искаженных додекаэдров $(\mathrm{La} / \mathrm{Ca}) \mathrm{O}_{8}$ и октаэдров $\mathrm{NbO}_{6}$, атомы $\mathrm{Li}$ частично занимают две кристаллографические позиции: тетраэдрическую $(24 d)$ и искаженную октаэдрическую $(96 h)$. Катионы лантана и кальция с отношением $2 / 3$ к $1 / 3$ занимают кристаллографическую позицию $(24 c)$ с симметрией $D_{2}$ (222). Кристаллические параметры твердых растворов и параметры уточнения рентгенограмм приведены в таблице. При замещении ионов $\mathrm{La}^{3+}\left(C R_{\mathrm{VIII}}\left(\mathrm{La}^{3+}\right)=1.160 \AA\right)$ ионами $\mathrm{Nd}^{3+}$ $\left(C R_{\mathrm{VIII}}\left(\mathrm{Nd}^{3+}\right)=1.109 \AA\right) \quad$ и $\quad \mathrm{Ho}^{3+} \quad\left(C R_{\mathrm{VIII}}\left(\mathrm{Ho}^{3+}\right)=\right.$ $=1.015 \AA)[21,22]$ наблюдается монотонное уменьшение объема элементарной ячейки.

Ранее по результатам КРС-исследований литиевых соединений со структурами тетрагонального и кубического граната $\mathrm{Li}_{5} \mathrm{La}_{3} M_{2} \mathrm{O}_{12}(M=\mathrm{Ta}, \mathrm{Hf}, \mathrm{Zr})$ было установлено, что полосы комбинационного рассеяния, наблюдаемые до $200 \mathrm{~cm}^{-1}$ и выше $600 \mathrm{~cm}^{-1}$, соответствуют колебаниям наиболее тяжелого катиона $\mathrm{La}$ и колебаниям

Параметры кристаллических решеток твердых растворов $\mathrm{Li}_{6} \mathrm{CaLa}_{2-x-y} \mathrm{Nd}_{x} \mathrm{Ho}_{y} \mathrm{Nb}_{2} \mathrm{O}_{12}\left(x=2.7 \cdot 10^{-5}-0.1 ; y=4 \cdot 10^{-8}\right.$ -0.05 ) (пространственная группа $I a-3 d, Z=8$ )

\begin{tabular}{l|c|c|c}
\hline \multicolumn{1}{c|}{$x$} & $y$ & $a, \AA$ & $V, \AA^{3}$ \\
\hline $2.7 \cdot 10^{-5} 5$ & $4 \cdot 10^{-8}$ & $12.72663(6)$ & $2061.30(2)$ \\
0.0125 & $9 \cdot 10^{-8}$ & $12.72644(5)$ & $2061.20(2)$ \\
0.025 & $1.4 \cdot 10^{-7}$ & $12.72577(5)$ & $2060.88(2)$ \\
0.05 & $2.4 \cdot 10^{-7}$ & $12.72397(7)$ & $2060.01(2)$ \\
0.1 & $4.5 \cdot 10^{-7}$ & $12.72046(6)$ & $2058.30(2)$ \\
0.025 & 0.025 & $12.72487(6)$ & $2060.44(2)$ \\
0.025 & 0.05 & $12.72464(6)$ & $2060.33(2)$
\end{tabular}


$M-\mathrm{O}$, соответственно [23-25]. Для нескольких литийлантановых оксидов и литиевых гранатов было показано, что колебания лития проявляются в диапазоне $200-600 \mathrm{~cm}^{-1}[26]$. Из-за сложного характера кристаллического строения литиевых гранатов можно ожидать значительное перекрывание внутренних мод $\mathrm{LiO}_{4}, \mathrm{LiO}_{6}$ и других координированных групп, присутствующих в структуре, и, следовательно, сложности в интерпретации спектров комбинационного рассеяния. Тем не менее, профиль КРС спектра $\mathrm{Li}_{6} \mathrm{CaLa}_{2} \mathrm{Nb}_{2} \mathrm{O}_{12}$ с минимальной концентрацией допантов близок к таковому для ку-

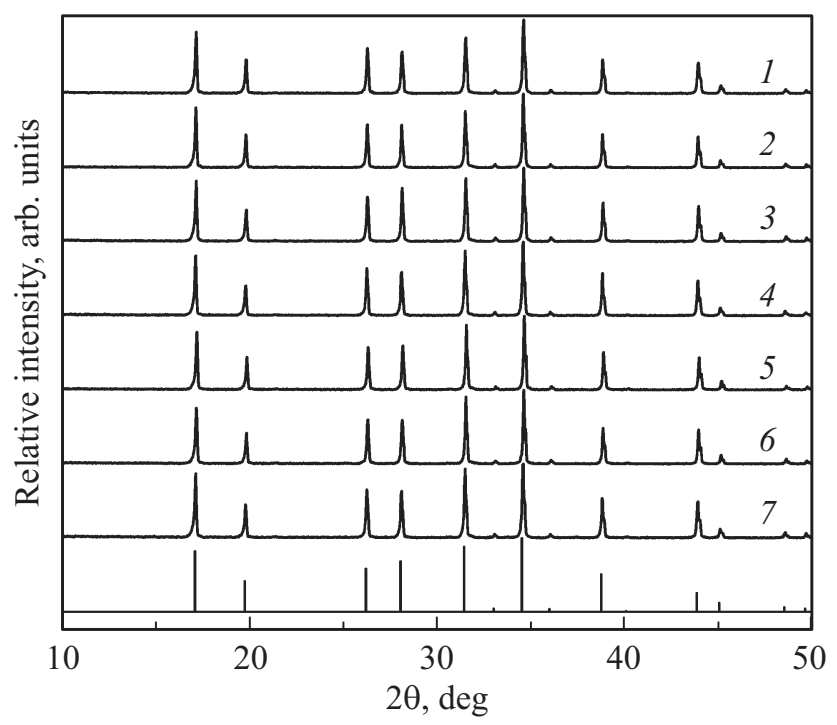

Рис. 1. Рентгенограммы твердых растворов $\mathrm{Li}_{6} \mathrm{CaLa}_{2-x-y} \mathrm{Nd}_{x} \mathrm{Ho}_{y} \mathrm{Nb}_{2} \mathrm{O}_{12} \quad$ при $\quad x=2.7 \cdot 10^{-5}$, $y=4 \cdot 10^{-8} \quad(1), x=0.0125, y=9 \cdot 10^{-8} \quad(2), x=0.025$, $y=1.4 \cdot 10^{-7} \quad(3), \quad x=0.05, \quad y=2.4 \cdot 10^{-7} \quad(4), x=0.1$, $y=4.5 \cdot 10^{-7}(5), x=0.025, y=0.025 \quad$ (6) и $x=0.025$, $y=0.05 \quad(7)$, вертикальные штрихи - положение разрешенных рефлексов $\mathrm{Li}_{6} \mathrm{CaLa}_{2} \mathrm{Nb}_{2} \mathrm{O}_{12}$ (FIZ ICSD card № 161386).

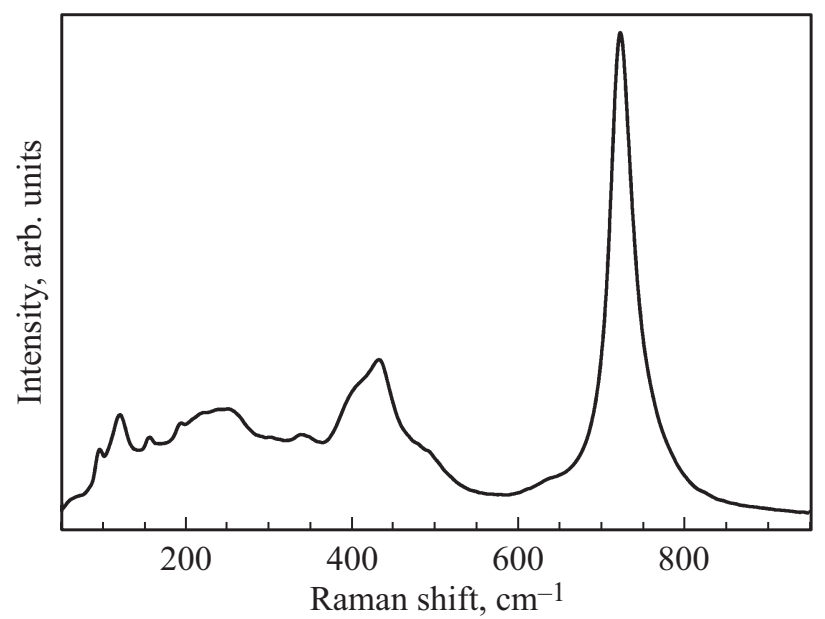

Рис. 2. Спектр комбинационного рассеяния кубического граната $\mathrm{Li}_{6} \mathrm{CaLa}_{2-x-y} \mathrm{Nd}_{x} \mathrm{Ho}_{y} \mathrm{Nb}_{2} \mathrm{O}_{12} \quad\left(x=2.7 \cdot 10^{-5}\right.$; $\left.y=4 \cdot 10^{-8}\right)$.

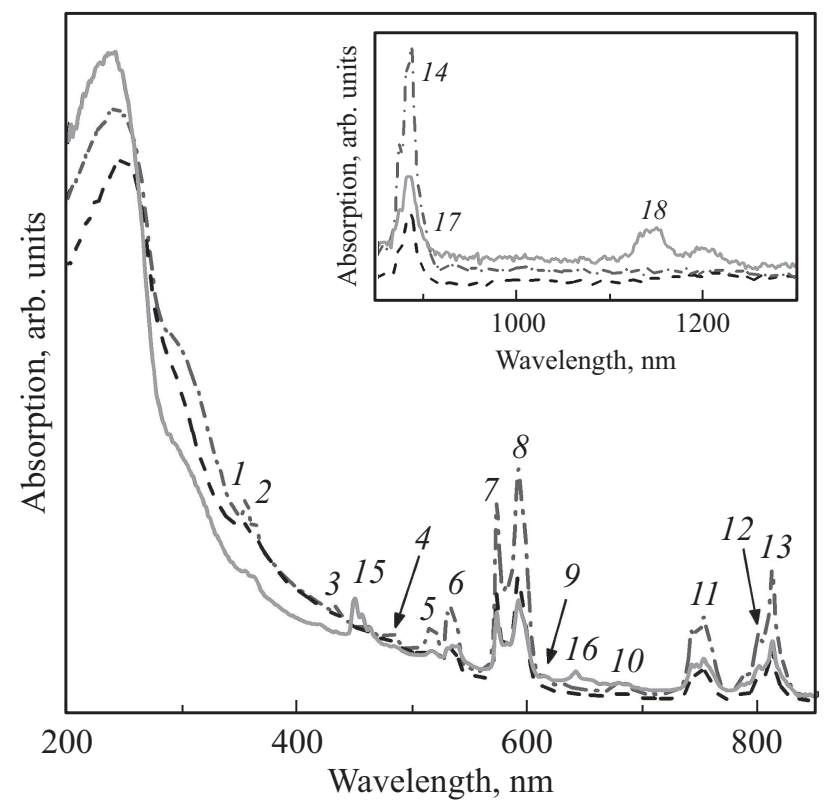

Рис. 3. Спектры поглощения соединений $\mathrm{Li}_{6} \mathrm{CaLa}_{2-x-y} \mathrm{Nd}_{x} \mathrm{Ho}_{y} \mathrm{Nb}_{2} \mathrm{O}_{12} \quad$ при $\quad x=0.1, \quad y=4.5 \cdot 10^{-7}$ (штрихпунктирная линия), $\quad x=0.025, \quad y=1.4 \cdot 10^{-7}$ (штриховая линия) и $x=0.025, y=0.05$ (сплошная линия) в диапазоне 200-850 nm. Вставка: спектры поглощения соединений в диапазоне $850-1300 \mathrm{~nm}$. Переходы: $\mathrm{Nd}^{3+}$ : ${ }^{4} I_{9 / 2} \rightarrow{ }^{2} L_{15 / 2},{ }^{4} D_{7 / 2}(1),{ }^{2} I_{11 / 2},{ }^{4} D_{1 / 2},{ }^{4} D_{5 / 2},{ }^{4} D_{3 / 2}(2),{ }^{2} P_{1 / 2}$, ${ }^{2} D_{5 / 2}$ (3), ${ }^{2} K_{15 / 2},{ }^{4} G_{11 / 2},{ }^{2} D_{3 / 2},{ }^{2} G_{9 / 2}$ (4), ${ }^{4} G_{9 / 2}$ (5), ${ }^{2} K_{13 / 2}$, ${ }^{4} G_{7 / 2}(6),{ }^{2} G_{7 / 2}(7),{ }^{4} G_{5 / 2}(8),{ }^{2} H_{11 / 2}$ (9), ${ }^{4} F_{9 / 2}$ (10), ${ }^{4} S_{3 / 2}$, ${ }^{4} F_{7 / 2}(11),{ }^{2} H_{9 / 2}(12),{ }^{4} F_{5 / 2}(13),{ }^{4} F_{3 / 2}(14), \mathrm{Ho}^{3+}:{ }^{5} I_{8} \rightarrow{ }^{5} G_{6}$, ${ }^{5} F_{1}(15),{ }^{5} I_{8} \rightarrow{ }^{5} F_{5}(16),{ }^{5} I_{8} \rightarrow{ }^{5} I_{5}(17),{ }^{5} I_{8} \rightarrow{ }^{5} I_{6}(18)$.

бического граната $\mathrm{Li}_{6} \mathrm{La}_{3} \mathrm{Ta}_{1.5} \mathrm{Y}_{0.5} \mathrm{O}_{12}$ (пространственная группа $I a-3 d, Z=8$ ) (рис. 2). Линии КРС в диапазоне $100-170 \mathrm{~cm}^{-1}$ и широкую линию с максимумом при $720 \mathrm{~cm}^{-1}$ следует отнести к валентным колебаниям додекаэдров $(\mathrm{La} / \mathrm{Ca}) \mathrm{O}_{8}$ и октаэдров $\mathrm{NbO}_{6}$, соответственно, а диапазон между 300 и $600 \mathrm{~cm}^{-1}$ приписать как внутренним колебаниям лития, так и деформационным колебаниям в октаэдре $\mathrm{NbO}_{6}$. Внутренние моды октаэдров $\mathrm{LiO}_{6}$ проявляются в диапазоне $200-300 \mathrm{~cm}^{-1}$, тогда как внутренние моды тетраэдров $\mathrm{LiO}_{4}$ наблюдаются в диапазоне $350-600 \mathrm{~cm}^{-1}$. Эффективная энергия фононов, вовлеченных преимущественно в процесс мультифононных безызлучательных переходов, равна или ниже энергии самой высокоэнергетичной колебательной моды. Таким образом, максимум фононной энергии составляет $\sim 720 \mathrm{~cm}^{-1}$, и, соответственно, кубический гранат $\mathrm{Li}_{6} \mathrm{CaLa}_{2} \mathrm{Nb}_{2} \mathrm{O}_{12}$ может быть рассмотрен в качестве перспективной оптической матрицы при создании материала для генерации излучения в ИК-диапазоне.

$\mathrm{Ha}$ рис. 3 приведены спектры поглощения $\mathrm{Li}_{6} \mathrm{CaLa}_{2-x-y} \mathrm{Nd}_{x} \mathrm{Ho}_{y} \mathrm{Nb}_{2} \mathrm{O}_{12} \quad\left(x=0.1, \quad y=4.5 \cdot 10^{-7}\right.$; $x=0.025, y=1.4 \cdot 10^{-7}$ и $\left.x=0.025, y=0.05\right)$, зарегистрированные в диапазоне длин волн $200-1350 \mathrm{~nm}$. Отмеченные на рис. 3 характерные полосы поглощения связаны с переходами $4 f-4 f$ из основного состояния на 
ряд возбужденных уровней ионов $\mathrm{Nd}^{3+}$ и $\mathrm{Ho}^{3+}$. Наличие интенсивной полосы поглощения около $808 \mathrm{~nm}$, обусловленной переходом ${ }^{4} I_{9 / 2} \rightarrow{ }^{4} F_{5 / 2}+{ }^{2} H_{9 / 2}$ в ионе $\mathrm{Nd}^{3+}$, свидетельствует о возможности использования для возбуждения излучения коммерческого лазерного диода. Как и следовало ожидать, интенсивность линий поглощения возрастает с увеличением концентрации ионов $\mathrm{Nd}^{3+}$ и $\mathrm{Ho}^{3+}$. Полосы, связанные с переходами в ионах $\mathrm{Ho}^{3+}$, не наблюдаются на спектрах поглощения соединений с низкой концентрацией гольмия. Спектр $\mathrm{Li}_{6} \mathrm{CaLa}_{1.925} \mathrm{Nd}_{0.025} \mathrm{Ho}_{0.05} \mathrm{Nb}_{2} \mathrm{O}_{12}$ содержит очень слабые линии при $\sim 450,650,900$ и $1150 \mathrm{~nm}$, соответствующие переходам на уровни $\left({ }^{5} G_{6},{ }^{5} F_{1}\right),{ }^{5} F_{5},{ }^{5} I_{5}$ и ${ }^{5} I_{6}$ иона $\mathrm{Ho}^{3+}$. Близость полос поглощения при 900 и $905 \mathrm{~nm}$, отнесенных к переходам ${ }^{4} I_{9 / 2} \rightarrow{ }^{4} F_{3 / 2}$ в $\mathrm{Nd}^{3+}$ и ${ }^{5} I_{8} \rightarrow{ }^{5} I_{5}$ в $\mathrm{Ho}^{3+}$, указывает на возможность использования ионов $\mathrm{Nd}^{3+}$ в качестве источника накачки ионов $\mathrm{Ho}^{3+}$.

Типичный спектр люминесценции твердых растворов $\quad \mathrm{Li}_{6} \mathrm{CaLa}_{2-x-y} \mathrm{Nd}_{x} \mathrm{Ho}_{y} \mathrm{Nb}_{2} \mathrm{O}_{12} \quad\left(x=2.7 \cdot 10^{-5}-0.1\right.$; $\left.y=4 \cdot 10^{-8}-0.05\right)$, записанный в диапазоне $1.0-3.0 \mu \mathrm{m}$ при возбуждении лазерным диодом с длиной волны $808 \mathrm{~nm}$, представлен на рис. 4, a. Спектр содержит эмиссионные полосы, соответствующие серии $4 f-4 f$-переходов в ионах неодима и гольмия. Наблюдаемое расщепление линий можно приписать к различным межуровневым переходам [27,28]. Линии в диапазоне длин волн $1.0-1.9 \mu \mathrm{m}$ связаны с переходами ${ }^{4} F_{3 / 2} \rightarrow{ }^{4} I_{11 / 2},{ }^{4} I_{13 / 2}$ и ${ }^{4} I_{15 / 2}$ в ионе $\mathrm{Nd}^{3+}$.
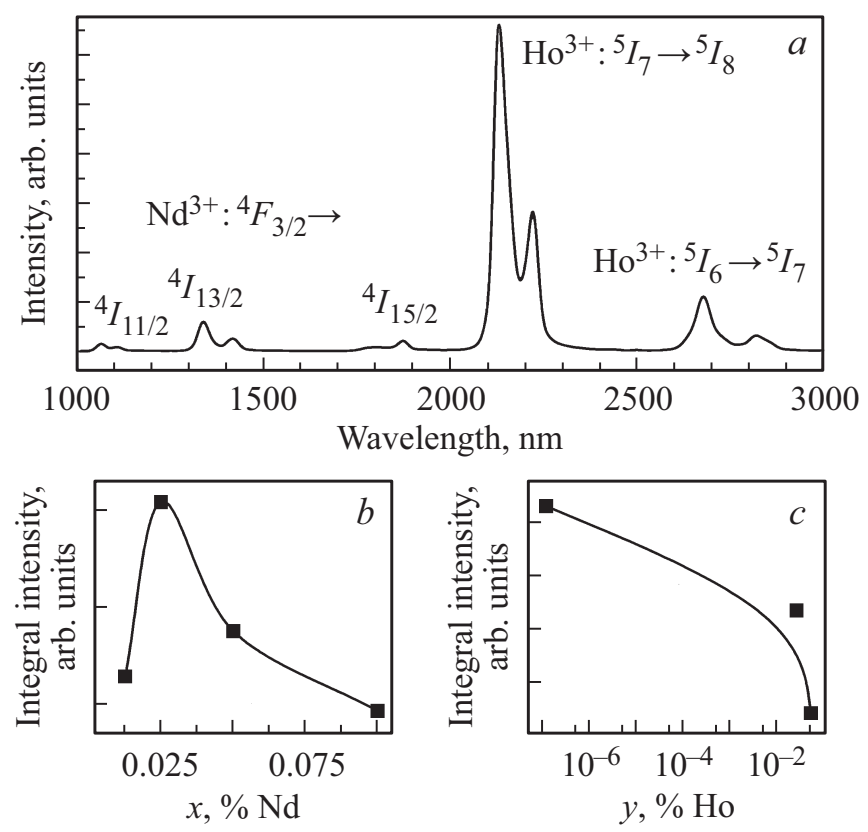

Рис. 4. Спектр люминесценции $\left(\lambda_{\mathrm{ex}}=808 \mathrm{~nm}\right)$ $\mathrm{Li}_{6} \mathrm{CaLa}_{1.975-y} \mathrm{Nd}_{0.025} \mathrm{Ho}_{y} \mathrm{Nb}_{2} \mathrm{O}_{12} \quad\left(y=1.4 \cdot 10^{-7}\right) \quad($ a), концентрационные зависимости интенсивности линии при $2.1 \mu \mathrm{m}$ от содержания неодима и гольмия в $\mathrm{Li}_{6} \mathrm{CaLa}_{2-x-y} \mathrm{Nd}_{x} \mathrm{Ho}_{y} \mathrm{Nb}_{2} \mathrm{O}_{12} \quad(x=0.0125-0.1 ; \quad y=$ $\left.=9 \cdot 10^{-8}-4.5 \cdot 10^{-7}\right)$ (b) и $\mathrm{Li}_{6} \mathrm{CaLa}_{1.975-y} \mathrm{Nd}_{0.025} \mathrm{Ho}_{y} \mathrm{Nb}_{2} \mathrm{O}_{12}$ $\left(y=1.4 \cdot 10^{-7}-0.05\right)(c)$.

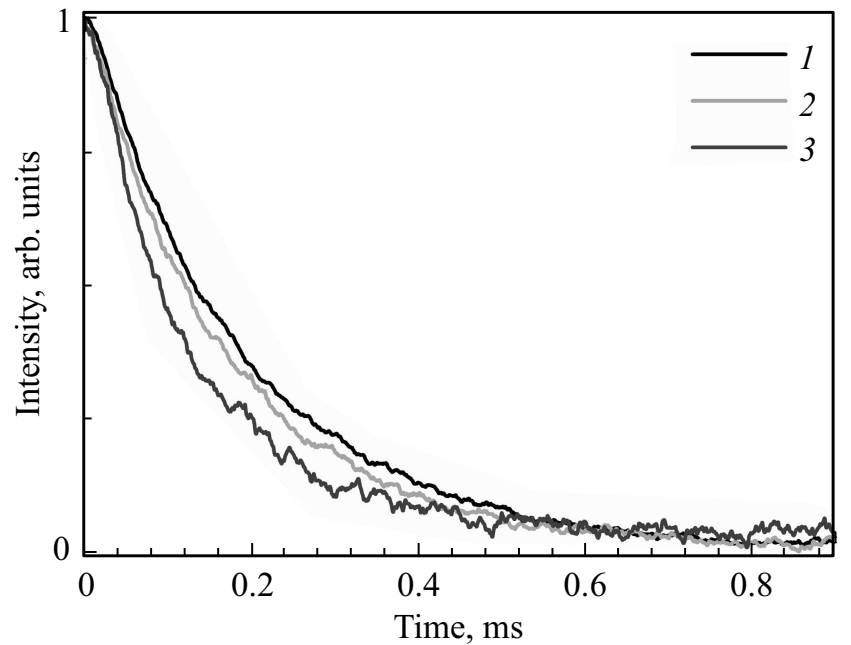

Рис. 5. Кривые затухания люминесценции $\left(\lambda_{\mathrm{ex}}=808 \mathrm{~nm}\right.$, $\left.\lambda_{\mathrm{em}}=2.1 \mu \mathrm{m}\right) \quad \mathrm{Li}_{6} \mathrm{CaLa}_{1.975-y} \mathrm{Nd}_{0.025} \mathrm{Ho}_{y} \mathrm{Nb}_{2} \mathrm{O}_{12} \quad$ при $y=1.4 \cdot 10^{-7}(1), y=0.025$ (2) и $y=0.05$ (3).

Из-за спектральных характеристик оптического фильтра ИКС7, линия при $0.9 \mu \mathrm{m}$, соответствующая переходу ${ }^{4} F_{3 / 2} \rightarrow{ }^{4} I_{9 / 2}$, не была зарегистрирована. К переходам ${ }^{5} I_{7} \rightarrow{ }^{5} I_{8} \quad$ и ${ }^{5} I_{6} \rightarrow{ }^{5} I_{7}$ в ионе Но $^{3+}$ следует отнести наиболее интенсивные линии с максимумами при 2.1 и $2.7 \mu \mathrm{m}$. Так как ионы гольмия содержатся главным образом в исходном реактиве оксида неодима, увеличение концентрации $\mathrm{Nd}^{3+}$ в $\mathrm{Li}_{6} \mathrm{CaLa}_{2-x-y} \mathrm{Nd}_{x} \mathrm{Ho}_{y} \mathrm{Nb}_{2} \mathrm{O}_{12}$ приводит к постепенному росту концентрации гольмия в образцах. Соответственно, интенсивность излучения увеличивается с содержанием $\mathrm{Nd}^{3+}$ до достижения максимума при $x=0.025$ и $y=1.4 \cdot 10^{-7}$ в $\mathrm{Li}_{6} \mathrm{CaLa}_{2-x-y} \mathrm{Nd}_{x} \mathrm{Ho}_{y} \mathrm{Nb}_{2} \mathrm{O}_{12}$ (рис. 4, b,c). Дальнейшее увеличение концентрации допантов приводит к уменьшению интенсивности люминесценции вследствие концентрационного тушения.

Кривые затухания люминесценции, зарегистрированные при длине волны $2.1 \mu \mathrm{m}$ при возбуждении лазерным диодом с длиной волны $808 \mathrm{~nm}$ для составов $\mathrm{Li}_{6} \mathrm{CaLa}_{1.975-y} \mathrm{Nd}_{0.025} \mathrm{Ho}_{y} \mathrm{Nb}_{2} \mathrm{O}_{12} \quad\left(y=1.4 \cdot 10^{-7}-0.05\right)$ демонстрируют экспоненциальный профиль (рис. 5). Величины времени жизни ${ }^{5} I_{7}$ уровня в ионах $\mathrm{Ho}^{3+}$ для этих соединений составляют 186, 164 и $124 \mu \mathrm{s}$ при $y=1.4 \cdot 10^{-7}, y=0.025$ и $y=0.05$, и близки к значениям, полученным для допированных гольмием фосфатов и германатов $[12,29,30]$.

Механизм многоступенчатых процессов переноса энергии, включающий участие ионов $\mathrm{Nd}^{3+}$ в качестве сенсибилизаторов инфракрасной люминесценции ионов $\mathrm{Ho}^{3+}$, представлен на рис. 6 и типичен для соединений, допированных неодимом и гольмием [30-33]. При поглощении излучения лазерного диода с длиной волны $808 \mathrm{~nm}$ происходит переход $\mathrm{Nd}^{3+}$ из основного состояния ${ }^{4} I_{9 / 2}$ в возбужденное состояние ${ }^{4} F_{5 / 2}+{ }^{2} H_{9 / 2}$ с последующим безызлучательным переходом на уровень ${ }^{4} F_{3 / 2}$. Дальнейшие переходы ${ }^{4} F_{3 / 2} \rightarrow{ }^{4} I_{9 / 2},{ }^{4} I_{11 / 2}$, 


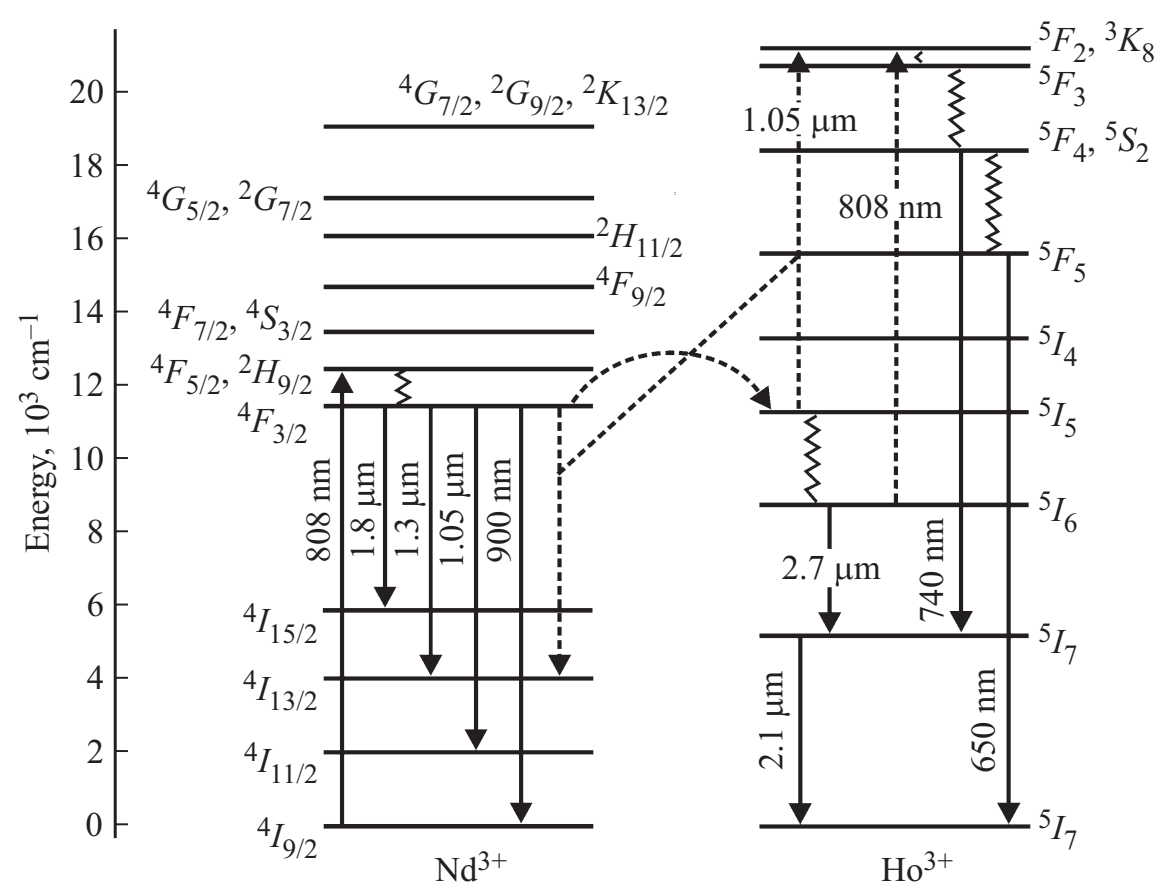

Рис. 6. Схема межуровневых переходов, возбуждения и излучения для ионов $\mathrm{Nd}^{3+}$ и $\mathrm{Ho}^{3+}$ в гранатах $\mathrm{Li}_{6} \mathrm{CaLa}_{2} \mathrm{Nb}_{2} \mathrm{O}_{12}: \mathrm{Nd}^{3+}, \mathrm{Ho}^{3+}$ $\left(\lambda_{\mathrm{ex}}=808 \mathrm{~nm}\right)$. Пунктирными линиями изображены процессы переноса энергии, мультифононной релаксации и кросс-релаксации, поглощения энергии возбужденным состоянием; непрерывными линиями - излучательные процессы.

${ }^{4} I_{13 / 2}$ и ${ }^{4} I_{15 / 2}$ сопровождаются появлением серии линий в ИК-диапазоне с максимумами при 0.90, 1.05, 1.30 и $1.80 \mu \mathrm{m}$ соответственно. Однако из-за близости энергии уровней ${ }^{4} F_{3 / 2}\left(\sim 11450 \mathrm{~cm}^{-1}\right)$ иона $\mathrm{Nd}^{3+}$ и ${ }^{4} I_{5}\left(\sim 11400 \mathrm{~cm}^{-1}\right)$ иона $\mathrm{Ho}^{3+}$ часто наблюдается процесс передачи энергии от донора $\left(\mathrm{Nd}^{3+}\right)$ к акцептоpy $\left(\mathrm{Ho}^{3}\right)$ по схеме: ${ }^{4} F_{3 / 2}\left(\mathrm{Nd}^{3+}\right)+{ }^{5} I_{8}\left(\mathrm{Ho}^{3+}\right) \rightarrow{ }^{4} I_{9 / 2}$ $\left(\mathrm{Nd}^{3+}\right)+{ }^{5} I_{5}\left(\mathrm{Ho}^{3+}\right)$. Последующий безызлучательный переход с уровня ${ }^{5} I_{5}$ приводит к заселению нижележащего уровня ${ }^{5} I_{6}$, с которого происходит переход на ${ }^{5} I_{7}$ состояние, сопровождающийся эмиссией при $2.7 \mu \mathrm{m}$. В случае соединений $\mathrm{Li}_{6} \mathrm{CaLa}_{2} \mathrm{Nd}_{x} \mathrm{Ho}_{y} \mathrm{Nb}_{2} \mathrm{O}_{12}$ наиболее интенсивная линия с максимумом при $2.1 \mu \mathrm{m}$ соответствует переходу ${ }^{5} I_{7} \rightarrow{ }^{5} I_{8}$ в ионах $\mathrm{Ho}^{3+}$.

Важно отметить, что для твердых растворов $\mathrm{Li}_{6} \mathrm{CaLa}_{2-x-y} \mathrm{Nd}_{x} \mathrm{Ho}_{y} \mathrm{Nb}_{2} \mathrm{O}_{12}$ соотношение интенсивностей линий в диапазоне $1.0-1.9 \mu \mathrm{m}$ существенно отличается от наблюдаемых в различных люминесцентных соединениях, активированных неодимом, для которых линия при $\sim 1.1 \mu \mathrm{m}$, соответствующая переходу $\mathrm{Nd}^{3+}: \quad{ }^{4} F_{3 / 2} \rightarrow{ }^{4} I_{11 / 2}, \quad$ является наиболее интенсивной $[28,34,35]$. Аномально низкому значению интенсивности данной линии в случаe $\mathrm{Li}_{6} \mathrm{CaLa}_{1.975-y} \mathrm{Nd}_{0.025} \mathrm{Ho}_{y} \mathrm{Nb}_{2} \mathrm{O}_{12}$, может способствовать процесс кросс-релаксации между ионами $\mathrm{Nd}^{3+}$ и $\mathrm{Ho}^{3+}$ по схеме: ${ }^{4} F_{3 / 2}\left(\mathrm{Nd}^{3+}\right)^{5} I_{5}\left(\mathrm{Ho}^{3+}\right) \rightarrow{ }^{4} I_{11 / 2}\left(\mathrm{Nd}^{3+}\right)+$ $+{ }^{5} F_{2},{ }^{3} K_{8}\left(\mathrm{Ho}^{3+}\right)$. В этом случае следует ожидать появление апконверси люминесценции в видимом диапазоне при возбуждении лазерным диодом с длиной волны $808 \mathrm{~nm}$.
Типичный спектр апконверсионной люминесценции в области длин волн 450-780 nm исследуемых соединений представлен на рис. 7. Линии с низкой интенсивностью при 650 и $750 \mathrm{~nm}$ соответствуют переходам ${ }^{5} F_{3} \rightarrow{ }^{5} I_{8} \quad$ и ${ }^{5} F_{4}, \quad{ }^{5} S_{2} \rightarrow{ }^{5} I_{8} \quad$ в ионе $\mathrm{Ho}^{3+}$. Подавление апконверсионных процессов в видимой спектральной области является необходимым условием для увеличения квантового выхода люминесценции в ИК-диапазоне [12,13]. Увеличение концентрации гольмия приводит лишь к незначительному росту интенсивности линий, и, соответственно, является подтвержде-

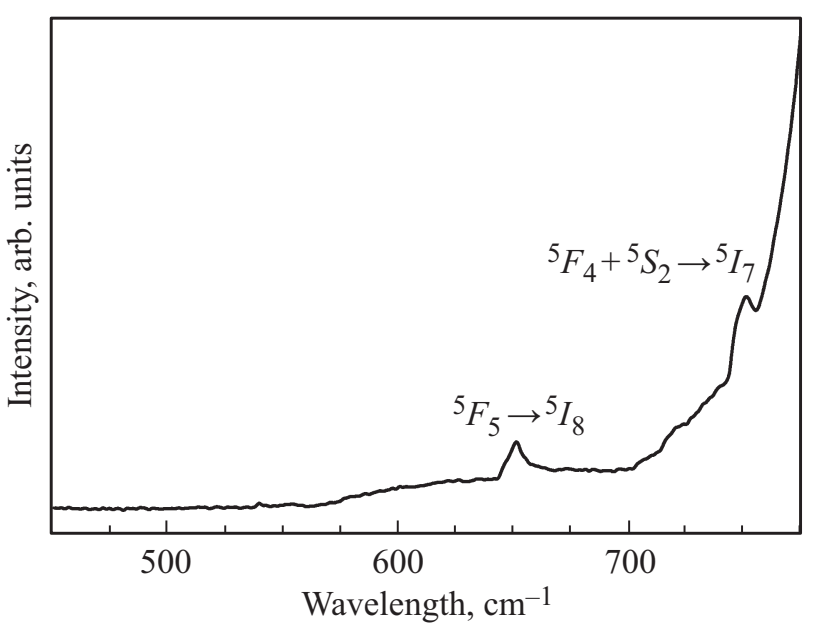

Рис. 7. Спектр апконверсионной люминесценции $\left(\lambda_{\text {ex }}=808 \mathrm{~nm}\right)$ соединения $\mathrm{Li}_{6} \mathrm{CaLa}_{1.875} \mathrm{Nd}_{0.025} \mathrm{Ho}_{0.05} \mathrm{Nb}_{2} \mathrm{O}_{12}$. 
нием эффективности данных соединений в качестве люминофоров коротковолнового ИК-диапазона. Вследствие слабой интенсивности линий, зависимость интенсивности апконверсионной люминесценции от подводимой мощности возбуждения, позволяющая определить число поглощенных ИК-фотонов, необходимых для излучения одного фотона в видимой области спектра, не может быть корректно измерена. Основываясь на расположении энергетических уровней иона гольмия и учитывая ранее полученные данные для германиевых соединений [12], следует ожидать протекание двухфотонного процесса. Таким образом, заполнение ${ }^{5} F_{2},{ }^{3} K_{8}$ уровня иона $\mathrm{Ho}^{3+}$ может происходить двумя различными путями: кросс-релаксационным процессом, по указанной выше схеме, и, в меньшей мере, за счет поглощения возбужденным состоянием в $\mathrm{Ho}^{3+}:{ }^{5} I_{5}\left(\mathrm{Ho}^{3+}\right)+h v \rightarrow{ }^{5} F_{2}$, ${ }^{3} K_{8} \quad\left(\mathrm{Ho}^{3+}\right)$ [31,36,37]. Соответственно, последующие безызлучательные переходы с уровня ${ }^{5} F_{2},{ }^{3} K_{8}$ приводят к заселению нижележащих уровней ${ }^{5} F_{4},{ }^{5} S_{2}$ и ${ }^{5} F_{5}$, с которых происходят переходы на ${ }^{5} I_{7}$ и основное ${ }^{5} I_{8}$ состояния, сопровождающиеся слабой эмиссией при 650 и $750 \mathrm{~nm}$, соответственно.

\section{4. Заключение}

Впервые синтезированы твердые растворы $\mathrm{Li}_{6} \mathrm{CaLa}_{2} \mathrm{Nb}_{2} \mathrm{O}_{12}: \mathrm{Nd}^{3+}, \mathrm{Ho}^{3+}$, исследованы спектральнолюминесцентные свойства и продемонстрирована вероятность использования данных соединений в качестве люминофоров ближнего и коротковолнового ИК-диапазона. Рентгенограммы соединений проиндицированы в кубической сингонии, пространственная группа $I a-3 d, Z=8$. Максимум фононной энергии по данным КРС составляет $\sim 720 \mathrm{~cm}^{-1}$. Спектры люминесценции ниобатов $\mathrm{Li}_{6} \mathrm{CaLa}_{2-x-y} \mathrm{Nd}_{x} \mathrm{Ho}_{y} \mathrm{Nb}_{2} \mathrm{O}_{12}$ в диапазоне $1.0-3.0 \mu \mathrm{m}$ содержат ряд линий, соответствующих характерным переходам в ионах $\mathrm{Nd}^{3+}$ и $\mathrm{Ho}^{3+}$. Следовые количества ионов гольмия и, соответственно, крайне низкое отношение допантов $\mathrm{Ho}^{3+}$ и $\mathrm{Nd}^{3+}$ позволяют получить интенсивную эмиссию в коротковолновой ИК-области спектра. Наибольшая интенсивность излучения достигается при $x=0.025$ и $y=4 \cdot 10^{-8}$ в $\mathrm{Li}_{6} \mathrm{CaLa}_{2-x-y} \mathrm{Nd}_{x} \mathrm{Ho}_{y} \mathrm{Nb}_{2} \mathrm{O}_{12}$. При возбуждении лазерным диодом с длиной волны $808 \mathrm{~nm}$ зарегистрирована слабая апконверсионная люминесценция в видимом диапазоне длин волн. Анализ полученных результатов позволяет сделать вывод о том, что в кубических гранатах $\mathrm{Li}_{6} \mathrm{CaLa}_{2} \mathrm{Nb}_{2} \mathrm{O}_{12}: \mathrm{Nd}^{3+}, \mathrm{Ho}^{3+}$ ионы $\mathrm{Nd}^{3+}$ выступают в качестве сенсибилизаторов ионов $\mathrm{Ho}^{3+}$, способствуя интенсивной коротковолновой ИК-люминесценции.

Рентгенографические исследования проведены в ЦКП „Рентгеноструктурный анализ“ при ИХТТ УрО РАН. Спектроскопия комбинационного рассеяния выполнена в ЦКП „Состав вещества“ при ИВТЭ УрО РАН. Я.В. Бакланова благодарит за поддержку СП-3310.2018.1.

\section{Список литературы}

[1] H. Kaushal, G. Kaddoum. IEEE Commun. Surv. Tut. 19, 57 (2017).

[2] N. Healy, U. Gibson, A.C. Peacock. Semicond. Sci. Technol. 33, 023001 (2018).

[3] F.K. Tittel, D. Richter, A. Fried. In: Solid-State MidInfrared Laser Sources / Ed. I.T. Sorokina, K.L. Vodopyanov. SpringerVerlag Berlin Heidelberg Ltd, Berlin. (2003). P. 458.

[4] A. Godard. C. R. Physique 8, 1100 (2007).

[5] K. Scholle, S. Lamrini, P. Koopmann, P. Fuhrberg. In: Frontiers in Guided Wave Optics and Optoelectronics / Ed. B.P. Pal. Intech Open Limited, London. (2010). P. 472.

[6] M. Pollnau, S.D. Jackson. In: Solid-State MidInfrared Laser Sources / Ed. I.T. Sorokina, K.L. Vodopyanov. Springer-Verlag Berlin Heidelberg Ltd, Berlin. (2003). P. 221.

[7] S.D. Jackson. Nat. Photonics, 6, 423 (2012); I.T. Sorokina, V.V. Dvoyrin, N. Tolstik, E. Sorokin. IEEE J. Sel. Top. Quantum Electron. 20, 090341 (2014).

[8] I.A. Khodasevich, A.S. Grabtchikov, A.A. Kornienko, E.B. Dunina. Opt. Spectrosc. 119, 759 (2015).

[9] I.A. Khodasevich, A.A. Kornienko, E.B. Dunina. A.S. Grabtchikov. J. Appl. Spectrosc. 81, 1056 (2015).

[10] О.А. Липина, Л.Л. Сурат, А.П. Тютюнник, В.Г. Зубков. Оптика и спектроскопия 121, 562 (2016).

[11] Y.V. Baklanova, A.N. Enyashin, L.G. Maksimova, A.P. Tyutyunnik, A.Yu. Chufarov, E.V. Gorbatov, I.V. Baklanova, V.G. Zubkov. Ceram. Int. 44, 6959 (2018).

[12] Y.V. Baklanova, O.A. Lipina, A.N. Enyashin, L.L. Surat, A.P. Tyutyunnik, N.V. Tarakina, A. Dominic Fortes, A.Yu. Chufarov, E.V. Gorbatov, V.G. Zubkov. Dalton Trans. 47, 14041 (2018).

[13] М.А. Мелкозерова, Я.В. Бакланова, О.А. Липина, А.Ю. Чуфаров, А.П. Тютюнник, В.Г. Зубков. ФТТ 60, 358 (2018).

[14] G. Chai, G. Dong, J. Qiu, Q. Zhang, Z. Yan. J. Phys. Chem. C 116, 19941 (2012).

[15] O.Ya. Manashirov, A.N. Georgobiani, V.B. Gutan, E.M. Zvereva, A.N. Lobanov. Inorg. Mater. 47, 1006 (2011).

[16] Y.V. Baklanova, O.A. Lipina, L.G. Maksimova, A.P. Tyutyunnik, I.I. Leonidov, T.A. Denisova, V.G. Zubkov. Spectrochim. Acta A 180, 105 (2017).

[17] G. Blasse, A.F. Corsmit. J. Solid State Chem. 6, 513 (1973).

[18] G. Blasse, M.J.J. Lammers, H.C.G. Verhaar, L.H. Brixner, C.C. Torardi. J. Solid State Chem. 60, 258 (1985).

[19] B.H. Toby. J. Appl. Crystallogr. 34, 210 (2001).

[20] A.C. Larson, R.B. Von Dreele. General Structure Analysis System (GSAS). Los Alamos, NM (2004). Los Alamos National Laboratory Report LAUR 86-748.

[21] R.D. Shannon, C.T. Prewitt. Acta Crystallogr. B 25, 925 (1969).

[22] Y.Q. Jia. J. Solid State Chem. 95, 184 (1991).

[23] L. Dhivya, R. Murugan. ACS Appl. Mater. Interfaces 6, 17606 (2014).

[24] S. Narayanan, G.T. Hitz, E.D. Wachsman, V. Thangaduraia. J. Electrochem. Soc. 162, A1772 (2015).

[25] I.I. Leonidov, Y.V. Baklanova, L.G. Maksimova, A.P. Tyutyunnik, L.A. Akashev, E.G. Vovkotrub, T.A. Denisova, V.G. Zubkov. J. Alloys Compd. 686, 204 (2016).

[26] G. Larraz, A. Orera, M.L. Sanjuan. J. Mater. Chem. A 1, 11419 (2013); [S. Narayana, G.T. Hitz, E.D. Wachsman, V. Thangaduraia. J. Electrochem. Soc. 162, A1772 2015.]

[27] J.B. Gruber, G.W. Burdick, U.V. Valiev, K.L. Nash, S.A. Rakhimov, D.K. Sardar. J. Appl. Phys. 106, 113110 (2009). 
[28] M. Pokhrel, N. Ray, G.A. Kumar, D.K. Sardar. Opt. Mater. Express 2, 235 (2012).

[29] Y. Tian, R. Xu, L. Hu, J. Zhang. J. Appl. Phys. 110, 033502 (2011).

[30] Y. Liu, M. Hu, Y. Wan, Z. You, J. Li, Z. Zhu, C. Tu. J. Lumin. 201, 143 (2018).

[31] J. Yuan, S.X. Shen, W.C. Wang, M.Y. Peng, Q.Y. Zhang, Z.H. Jiang. J. Appl. Phys. 114, 133506 (2013).

[32] J. Yuan, S.X. Shen, D.D. Chen, Q. Qian, M.Y. Peng, Q.Y. Zhang. J. Appl. Phys. 113, 173507 (2013).

[33] L.X. Li, W.C. Wang, C.F. Zhang, J. Yuan, B. Zhou, Q.Y. Zhang. Opt. Mater. Express 5, 2904 (2016).

[34] M.J. Weber, T.E. Varitimos. J. Appl. Phys. 42, 499 (1971).

[35] J.W. Stouwdam, F.C.J.M. Veggel. Nano Lett. 2, 733 (2002).

[36] N.K. Giri, D.K. Rai, S.B. Rai. Appl. Phys. 104, 113107 (2008).

[37] R.S. Yadav, R.V. Yadav, A. Bahadur, S.B. Rai. RSC Adv. 6, 51768 (2016).

Редактор Т.Н. Василевская 